\title{
Prevalence and Determinants of Anogenital Colonization by Group B Streptococcus Infection Among HIV Positive and Negative Women in Calabar, Nigeria
}

\author{
Charles Njoku$^{1}$, Cajethan Emechebe ${ }^{1 *}$, Anthony Agbakwuru ${ }^{1}$
}

\begin{abstract}
Objectives: Despite significant global decline in neonatal mortality, the rates are still unacceptably high in sub-Saharan African countries. One of the common and preventable causes of neonatal mortality is neonatal infection with group B streptococcus (GBS) microorganism. This study aims to determine the prevalence of anogenital colonization by GBS bacteria among HIV positive women, factors influencing colonization and the antimicrobial sensitivity in women attending antenatal clinic in our center.

Materials and Methods: This was a prospective study conducted at the antenatal clinics of University of Calabar Teaching Hospital (UCTH), Calabar. A total of 84 eligible and consented HIV positive women and 84 HIV negative women that were within 35 to 37 weeks of gestational age matched for age and parity were studied.

Results: Eighteen subjects tested positive to GBS infection with overall prevalence of $10.7 \%$. However, 13 (15.5\%) subjects with HIV infection tested positive to GBS infection and that was significantly higher compared with 5 (6.0\%) among women without HIV infection. The prevalence of GBS infection was significantly higher among subjects with primary education (54.5\%). Among HIV positive subject, there was significantly higher prevalence of GBS infection among concordant couples compared to discordant couples $(P=0.04)$. Most of the subjects were sensitive to ceftriaxone $(88.9 \%)$ and erythromycin $(72.2 \%)$, and drug sensitivity was least with ampicillin (16.7\%).

Conclusion: Anogenital colonization with GBS is high among pregnant women in our center and significantly higher among HIV infected subjects. Preventive approach to GBS colonization is a worthy measure and there is need to institute GBS screening among high risk pregnancies such as HIV infected women.

Keywords: Group B streptococcus, HIV, Neonatal mortality, Antimicrobial sensitivity, Calabar
\end{abstract}

\section{Introduction}

Sub-Saharan African region still contributes the highest proportion of the global burden of neonatal mortality due to infections (1). Many neonates are still dying of preventable causes of deaths including vertical transmission of Group B streptococcus (GBS) (2). Consequently, GBS infection remains an important cause of perinatal morbidity and mortality including neonatal meningitis, pneumonia and sepsis (3). It is also responsible for significant maternal peripartum diseases such as chorioamnionitis, endometritis and urinary tract infections (4). Cervicitis, preterm premature ruptures of membranes, preterm labor and stillbirth have also been reported among GBS colonized mothers. It is also a known cause of several infectious diseases in older children, women, immune-compromised patients, and the elderly (5). Hence, maternal infection and subsequent vertical transmission to their infants is a significant cause of maternal and neonatal morbidity and mortality.

GBS is a gram-positive bacterium which is widely distributed in nature and a normal flora of the gastrointestinal and female genital tracts. The neonate is at greater risk of GBS infection upon delivery and premature babies are at greatest risk of death and disease (6). Most frequently the neonate becomes infected with GBS during labor through vertical transmission from the GBS colonized mother. Vertical transmission of GBS from colonized mother can result in $50 \%$ to $75 \%$ of their neonates becoming colonized with GBS. About $1 \%$ to $2 \%$ of these infants, who acquire GBS from their mothers, will develop invasive disease, with case fatality rate of $4 \%$ to $10 \%(7,8)$.

Neonatal septicemia due to GBS infection could either occur within 7 days postpartum which is termed early onset disease (EOD) which accounts for about $80 \%$ of GBS neonatal infections or between 7 days and 90 days postpartum which is termed late onset disease (LOD) which accounts for the remaining 20\%. During labor, a healthy mother colonized with GBS does not always show signs or symptoms of colonization and the disease (GBS) is only occasionally associated with urinary tract infection; therefore vertical transmission from mother to neonate during delivery may occur unnoticed and result in neonatal disease (9). The occasional asymptomatic nature 
of GBS infection and consequent transmission to the neonates emphasizes the importance of screening of these women between 35 to 37 weeks of gestation (9). Maternal detection of GBS during pregnancy and administration of treatment to the mother during labor will lead to decreased incidence of neonatal GBS colonization and subsequently a reduction in the incidence of neonatal GBS disease. The Centers for Disease Control and Prevention (CDC) revised guideline recommended universal screening for GBS for all pregnant women from 35 to 37 weeks of gestation (7). Despite these measures, GBS is a main cause of infectious mortality and morbidity among newborns (10).

In a study done in Brazil, 19.8\% of HIV infected pregnant women between 35 to 37 weeks gestation were found to be GBS colonized, which is higher than the prevalence of $14.1 \%$ in the control group without HIV. A similar pattern of the disease was shown in a study in Southern Africa (11). A cross-sectional study of GBS colonization on 509 pregnant women in the DRC found $50 \%$ prevalence among HIV positive women which is significantly higher than $23.7 \%$ among HIV negative subjects (12). However studies conducted in Uganda and Malawi showed that there was no significant association with HIV status $(3,13)$.

It is obvious that no consensus has been reached concerning association of HIV infection with GBS colonization. The introduction of highly active antiretroviral drugs has markedly increased the survival rate of HIV infected women and it is important to determine whether this group of women has more predispositions to acquisition of GBS infection. Therefore, this work was designed to evaluate the association between GBS and HIV infection in pregnancy. The findings may suggest preventive measures such as antenatal screening, intrapartum antibiotic prophylaxis and possible GBS vaccination in condition of high prevalence in the region under study. Despite high clinical significance of GBS infection, there is a paucity of literature on the epidemiology of GBS infection among pregnant women in our locality.

\section{Materials and Methods}

This was a prospective comparative study conducted at the antenatal care clinics of University of Calabar Teaching Hospital (UCTH) over a period of 16 weeks from March 1, 2016 to June 31, 2016. UCTH is a tertiary healthcare facility which serves as the main referral center for Cross River state with estimated population of 2888966 people (14).

\section{Inclusion Criteria}

The inclusion criteria for cases were consented pregnant women who were confirmed HIV positive and were within 35 to 37 weeks gestational age while the control group subjects were consented HIV negative pregnant women within 35 to 37 weeks of gestation.

\section{Exclusion Criteria}

Non-consenting pregnant women were excluded from the study and any pregnant women that received any form of antibiotic therapy within 2 weeks prior to commencement of the study were also excluded.

\section{Sample collection}

For each HIV positive subjects that was recruited, a socio-demographically similar HIV negative pregnant woman was recruited by simple random sampling. Sociodemographic similarity by age, marital status, and parity were chosen. Eligible pregnant women that consented to participate were counseled on the objectives and benefits of the study, and available treatment options. Recruitment was followed by data collection of sociodemographic characteristics, obstetric history and collection of laboratory samples for assessment of GBS colonization and sensitivity testing. Patients were kept in dorsal position with both knees flexed, observing routine aseptic procedure, with gloved hands, patients' anogenital samples were collected as follows: the labia minora were parted, a sterile swab stick was inserted up to 2 to $3 \mathrm{~cm}$ into the vagina and vaginal wall was swabbed circumferentially. A separate swab was used to swab the anus at the level of the anal sphincter. Swabs specimen collected were transported to the hospital Microbiology Research laboratory using Amies transport medium after proper labeling of specimen for analysis. After collecting the swabs from the vagina and anus, culture of the specimen was done within one hour of collection. The analysis of the specimen was done by a microbiologist and a laboratory scientist who was designated for the study. Isolates which showed characteristic morphology (small $(0.5$ to $1 \mathrm{~mm})$, round, domed, smooth surfaced, translucent, mildly $\beta$-hemolytic or $\gamma$-hemolytic, entire edged colonies) on sheep blood agar were presumptively identified as Streptococcus agalactiae. Colonies with or without narrow zone of hemolysis were gram stained. To distinguish between streptococci and staphylococci, enzyme catalase test was done. GBS was identified as nonmotile, gram positive and catalase-negative cocci. The absence of effervescence was recorded as catalase negative which is a characteristic of GBSs.

\section{Analysis of Data}

Data was entered and analyzed using SPSS version 20.0. Frequency distributions of socio-demographic and obstetric characteristics of subjects were presented on frequency tables and charts. The prevalence or proportion of subjects with positive GBS test results was presented as total prevalence. Chi-square test and Fisher exact $P$ value were used to compare categorical variable while independent $t$ tests was used to compare means of the variables in the assessment of risk factors for GBS colonization. The results were presented in tables and graph. $P$ values less than 0.05 were considered statistically significant at $95 \% \mathrm{CI}$.

\section{Results}

A total of 168 subjects were surveyed, from equal 
proportion of two groups of pregnant women with and without HIV infection. From this overall number, 18 subjects tested positive to GBS infection yielding an overall prevalence of $10.7 \%$. The prevalence of GBS infection among HIV infected women was $15.5 \%$ while it was $6.0 \%$ among HIV negative women.

The prevalence of GBS infection was significantly higher among the subjects with primary (54.5\%), compared with secondary $(8.6 \%)$ and tertiary $(6.6 \%)$ levels of education $(P=0.00)$ as shown in Table 1 . Other socio-demographic factors, including age groups, occupation, tribe, residential location and alcohol consumption did not show significant differences in prevalence of GBS infection $(P>0.05)$.

There was no significant difference in mean values of various anthropometric and obstetric factors assessed comparing the subjects with and without GBS infection
(Table 2).

Subjects with HIV infection had significantly higher prevalence of GBS infection, compared with those without HIV infection ( $15.5 \%$ vs. $6.0, P=0.04$ ) as shown in Table 3. Also, among HIV positive subjects, there was significantly higher prevalence of GBS infection among concordant couples compared with discordant couples (29.6 vs. $8.3 \%$, $P=0.04)$. Other obstetric factors including parity, use of contraception and last period of unprotected sex, did not show significant differences in the prevalence of GBS infection.

Figure 1 shows a bar chart of Drug sensitivity to GBS. Among the 18 pregnant women who tested positive to GBS infection, most subjects were sensitive to ceftriaxone $16(88.9 \%)$ and erythromycin $13(72.2 \%)$, and drug sensitivity was least with ampicillin $3(16.7 \%)$ and

Table 1. Socio-Demographic Characteristics of the Women and Associated With GBS Infection $(\mathrm{N}=168)$

\begin{tabular}{|c|c|c|c|c|}
\hline Variable & $\begin{array}{c}\text { GBS Present } \\
\text { No. (\%) }\end{array}$ & $\begin{array}{c}\text { GBS Absent } \\
\text { No. (\%) }\end{array}$ & $\begin{array}{c}\text { Total } \\
\text { No. }(100)\end{array}$ & $P$ Value \\
\hline \multicolumn{5}{|l|}{ Age group (y) } \\
\hline$<20$ & $0(0)$ & $8(100)$ & $8(100)$ & \multirow[t]{6}{*}{0.73} \\
\hline $21-25$ & $2(5.6)$ & $34(94.4)$ & $36(100)$ & \\
\hline $26-30$ & $8(13.3)$ & $52(86.7)$ & $60(100)$ & \\
\hline $31-35$ & $6(12.2)$ & $43(87.8)$ & $49(100)$ & \\
\hline $36-40$ & $2(14.3)$ & $12(85.7)$ & $14(100)$ & \\
\hline$>40$ & $0(0)$ & $1(100)$ & $1(100)$ & \\
\hline \multicolumn{5}{|c|}{ Highest educational level } \\
\hline Primary & $6(54.5)$ & $5(45.5)$ & $11(100)$ & \multirow[t]{3}{*}{0.00} \\
\hline Secondary & $7(8.6)$ & 74 (91.4) & $81(100)$ & \\
\hline Tertiary & $5(6.6)$ & 71 (93.4) & $76(100)$ & \\
\hline \multicolumn{5}{|l|}{ Occupation } \\
\hline Trader & $5(9.8)$ & $46(90.2)$ & $51(100)$ & \multirow[t]{7}{*}{0.92} \\
\hline Civil servant & $4(15.4)$ & $22(84.6)$ & $26(100)$ & \\
\hline Farmer & $4(12.1)$ & 29 (87.9) & $33(100)$ & \\
\hline Student & $1(6.7)$ & $14(93.3)$ & $15(100)$ & \\
\hline House wife & $0(0)$ & $8(100)$ & $8(100)$ & \\
\hline Artisan & $3(12.5)$ & $21(87.5)$ & $24(100)$ & \\
\hline Unemployed & $1(9.1)$ & $10(90.9)$ & $11(100)$ & \\
\hline \multicolumn{5}{|l|}{ Tribe } \\
\hline Ibibio/Annang & $6(15.4)$ & $33(84.6)$ & 39 (100) & \multirow[t]{6}{*}{0.75} \\
\hline Efik & $4(14.8)$ & $23(85.2)$ & $27(100)$ & \\
\hline Ibo & $2(8.0)$ & $23(92.0)$ & $25(100)$ & \\
\hline Ekoi & $1(4.3)$ & $22(95.7)$ & $23(100)$ & \\
\hline Ejagham & $2(9.1)$ & 20 (90.9) & $22(100)$ & \\
\hline Others & $3(9.4)$ & $29(90.6)$ & $32(100)$ & \\
\hline \multicolumn{5}{|c|}{ Location of subject } \\
\hline Urban & $7(8.3)$ & $77(91.7)$ & $84(100)$ & \multirow[t]{2}{*}{0.32} \\
\hline Rural & $11(13.1)$ & $73(86.9)$ & $84(100)$ & \\
\hline \multicolumn{5}{|c|}{ Alcohol consumption } \\
\hline Yes & $4(10.8)$ & $33(89.2)$ & $37(100)$ & \multirow[t]{2}{*}{0.98} \\
\hline No & $14(10.7)$ & $117(89.3)$ & $131(100)$ & \\
\hline
\end{tabular}

Abbreviation: GBS, group B streptococcus. 
Table 2. Anthropometric and Obstetric Factors Associated With GBS Infection ( $N=168)$

\begin{tabular}{|c|c|c|c|c|}
\hline Variable & GBS Present, No. (\%) & GBS Absent, No. (\%) & $T$ test & $P$ Value \\
\hline \multicolumn{5}{|l|}{ Age } \\
\hline Mean (SD) & 30.4 (3.9) & $28.8(4.7)$ & 1.4 & 0.15 \\
\hline Range & $22-38$ & $18-41$ & & \\
\hline \multicolumn{5}{|l|}{ BMI } \\
\hline Mean (SD) & $29.0(5.0)$ & $28.8(4.9)$ & 0.14 & 0.89 \\
\hline Range & $23.4-43.6$ & $20.1-44.4$ & & \\
\hline \multicolumn{5}{|c|}{ ART duration $(y)(n=84)$} \\
\hline Mean (SD) & $3.4(2.1)$ & $3.7(2.9)$ & 0.23 & 0.82 \\
\hline Range & $2-7$ & $1-16$ & & \\
\hline \multicolumn{5}{|c|}{ CD4 count (cells/mL) $(\mathrm{n}=84)$} \\
\hline Mean (SD) & 434 (198) & $530(252)$ & 1.16 & 0.25 \\
\hline Range & $118-877$ & $180-1227$ & & \\
\hline \multicolumn{5}{|c|}{ GA at booking (wk) } \\
\hline Mean (SD) & $22.0(7.7)$ & $21.3(7.2)$ & 0.39 & 0.70 \\
\hline Range & 8-34 & $8-36$ & & \\
\hline \multicolumn{5}{|c|}{ GA at delivery (wk) } \\
\hline Mean (SD) & $37.6(1.6)$ & $37.9(1.5)$ & 0.87 & 0.39 \\
\hline Range & $35-40$ & $35-41$ & & \\
\hline \multicolumn{5}{|l|}{ Parity } \\
\hline Mean (SD) & $1.5(1.3)$ & $1.5(1.2)$ & 0.02 & 0.98 \\
\hline Range & $0-5$ & $0-6$ & & \\
\hline \multicolumn{5}{|l|}{ Gravidity } \\
\hline Mean (SD) & $2.6(1.4)$ & $2.7(1.3)$ & 0.25 & 0.80 \\
\hline Range & $1-6$ & $1-7$ & & \\
\hline \multicolumn{5}{|c|}{ Number of children } \\
\hline Mean (SD) & $1.5(1.4)$ & $1.5(1.2)$ & 0.04 & 0.97 \\
\hline Range & $0-5$ & $0-5$ & & \\
\hline
\end{tabular}

Abbreviations: GBS, group B streptococcus; GA, gestational age; ART, anti-retroviral therapy; SD, standard deviation.

Table 3. Retroviral and Obstetric Factors Associated With GBS Infection $(N=168)$

\begin{tabular}{|c|c|c|c|c|}
\hline Variable & GBS Present, No. (\%) & GBS Absent, No. (\%) & Total No. (100) & Fisher Exact $\boldsymbol{P}$ Value \\
\hline \multicolumn{5}{|l|}{ Retroviral status } \\
\hline Positive & $13(15.5)$ & $71(84.5)$ & $84(100)$ & 0.04 \\
\hline Negative & $5(6.0)$ & $79(94.0)$ & $84(100)$ & \\
\hline \multicolumn{5}{|l|}{ Partner HIV status $(\mathrm{n}=84$ ) } \\
\hline Discordant & $2(8.3)$ & $22(91.7)$ & $24(100)$ & 0.04 \\
\hline Concordant & $16(29.6)$ & $38(70.4)$ & $54(100)$ & \\
\hline Unknown & $3(50.0)$ & $3(50.0)$ & $6(100)$ & \\
\hline \multicolumn{5}{|c|}{ Time of HIV diagnosis $(n=84)$} \\
\hline Before pregnancy & $8(15.7)$ & $43(84.3)$ & $51(100)$ & 0.94 \\
\hline During index pregnancy & $5(15.2)$ & $28(84.8)$ & $33(100)$ & \\
\hline \multicolumn{5}{|l|}{ Receiving ART $(n=84)$} \\
\hline Yes & $9(12.5)$ & $63(87.5)$ & $72(100)$ & 0.07 \\
\hline No & $4(33.3)$ & $8(66.7)$ & $12(100)$ & \\
\hline \multicolumn{5}{|l|}{ Parity category } \\
\hline Nulliparous & 5 (13.9) & $31(86.1)$ & $36(100)$ & 0.49 \\
\hline Multiparous & $13(9.8)$ & $119(90.2)$ & $132(100)$ & \\
\hline \multicolumn{5}{|l|}{ Previous contraceptive use } \\
\hline Yes & $3(20.0)$ & $12(80.0)$ & $15(100)$ & 0.22 \\
\hline No & $15(9.8)$ & $138(90.2)$ & $153(100)$ & \\
\hline \multicolumn{5}{|l|}{ Last period unprotected sex } \\
\hline Within last 3 days & $2(4.4)$ & $43(95.6)$ & $45(100)$ & 0.47 \\
\hline 4-7 days & 5 (13.9) & $31(86.1)$ & $36(100)$ & \\
\hline 8-28 days & 7 (15.9) & $37(84.1)$ & $44(100)$ & \\
\hline 28 days to 3 months & $2(9.5)$ & $19(90.5)$ & $21(100)$ & \\
\hline Beyond 3 months & $2(9.1)$ & $20(90.9)$ & $22(100)$ & \\
\hline
\end{tabular}

Abbreviations: GBS, group B streptococcus; ART, anti-retroviral therapy 


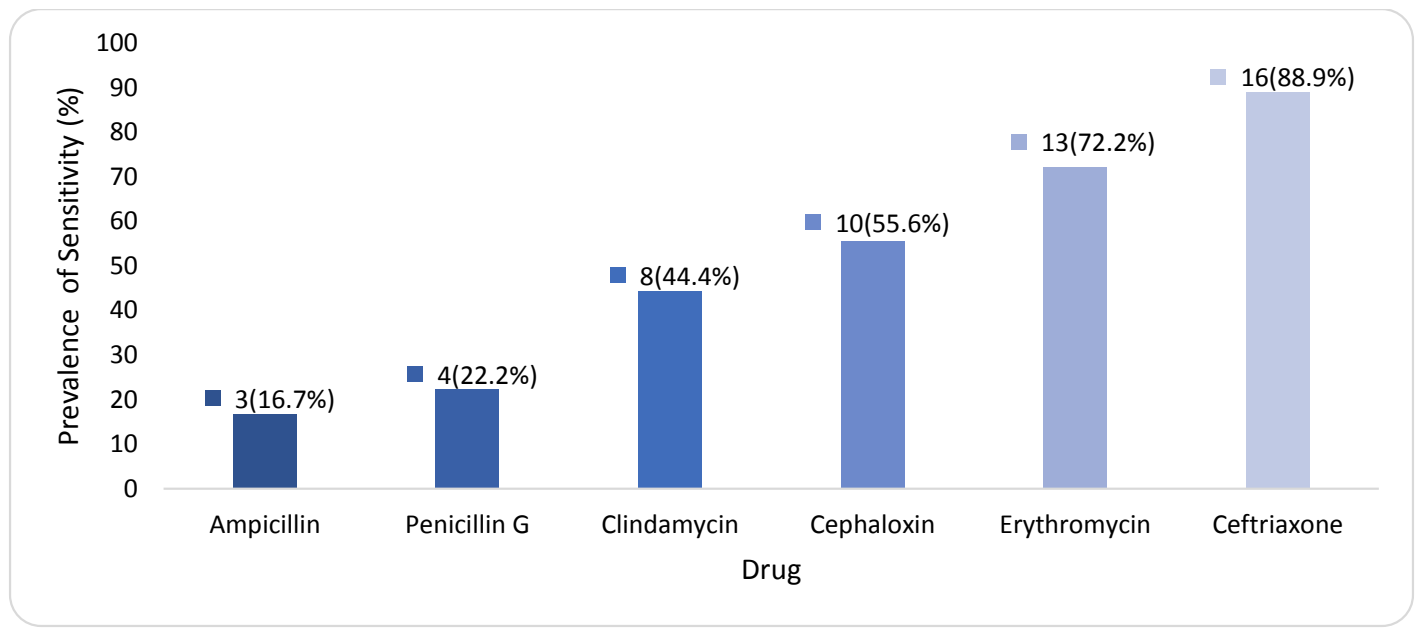

Figure 1. The Prevalence of Drug Sensitivity to GBS.

penicillin G $4(22.2 \%)$.

\section{Discussion}

Overall prevalence of anogenital GBS colonization in this study was $10.7 \%$. This is similar to $9 \%$ prevalence previously reported in Calabar, the same study setting (15), and $11.3 \%$ reported in Ile-Ife, Nigeria (16). Comparable prevalence rates of $10 \%$ and $9.8 \%$ were also reported in similar studies in Ibadan (17) and Maiduguri (18), Nigeria, respectively. However, much lower prevalence rate of $1.33 \%$ was reported in Uyo (19) and 6.6\% was reported in Jos, Nigeria (20). On the contrary, higher prevalence rates of $14 \%, 28.8 \%$ and $21.61 \%$ have been reported in earlier studies in Zaria, Nigeria (21), Uganda (22) and South Africa (23), respectively. A study in the DRC reported overall colonization rate of $20 \%$ amongst pregnant women (12). Outside Africa, low colonization rates were reported in places such as Greece (6.6\%) (24) and India (9.66\%) (25). The disparity in prevalence reported in the various studies agrees with possible roles played by geographical, environmental, and genetic factors and sampling technique in GBS prevalence in the various study settings (26). Disparity in prevalence may also be due to differences in GBS identification techniques used, with possible higher sensitivity of detection using Lancefield antigen following culture enrichment rather than direct culture (27). In this study, anal and vaginal swabs were separately collected, cultured and analyzed which is expected to have increased sensitivity to GBS. Further studies are required to identify specific aspects of lifestyle, behavior or cultural practices that may be responsible for increase in prevalence of GBS in different geographical locations.

In this study, prevalence of anogenital colonization with GBS among HIV infected subjects was found to be higher compared with non-infected subjects, and this was statistically significant ( $15 \%$ vs. $6.0 \%, P=0.04)$. Our result agrees with a recent study in DRC where the prevalence of GBS in pregnant women was significantly higher among HIV positive women compared with HIV negative women. However, such an association was not found in other previous studies $(28,29)$. A similar study in Malawi showed no significant difference in prevalence of GBS infection among HIV infected and non-infected subjects $(21.7 \%$ vs. $19.4 \%, P=0.32)$ (28).In addition, in California there was no significant association between GBS colonization and HIV status (29). The reasons for these discrepancies remain unclear. Implication of HIV infection as possible enabling factor for GBS infection may be based on immunosuppressive effect of retroviral disease, which increases susceptibility to infections. This may however be difficult to substantiate in this study where the mean CD4 count was not found to be significantly different comparing those with and without GBS infection. Similar study in Malawi found no significant difference in prevalence of GBS colonization among HIV positive women that had higher CD4 count levels (28).

In this study, level of education was significantly associated with GBS infection and was significantly higher among the subjects with primary (54.5\%), compared with secondary $(8.6 \%)$ and tertiary (6.6\%) levels of education. Level of education may be associated with lower socioeconomic status, poor personal and environmental hygiene and poor access to preventive health services. This probably may not be unconnected to the difference in the level of hygiene and readiness to access health care demonstrable by the subjects with different levels of education. Other socio-demographic factors such as age, occupation and alcohol consumption were not significantly associated with GBS infection, suggesting that they are not risk factors that determine infectivity with GBS infection in the study setting.

None of the anthropometric and obstetric factors were found to be significantly associated with GBS infection. This includes mean CD4 count, which suggest that CD4 count may not be the sole determinant of the level of immunity, hence may not be a significant determinant of GBS infection. This is especially so when other factors 
may determine level of immunity, including nutrition, stress and genetics. Similarly, duration on ART was not found to be significantly different comparing subjects with and without GBS infection.

Most infections with GBS in the study area were sensitive to ceftriaxone (88.9\%) and erythromycin $(72.2 \%)$, and drug sensitivity was least with ampicillin (16.7\%) and penicillin $\mathrm{G}(22.2 \%)$. There was no difference in drug sensitivity comparing the subject with and without HIV infection. A previous study in the same setting (Calabar), found sensitivity to ampicillin (100\%) and penicillin G (100\%) (15), while in different setting found resistance to ampicillin (100\%) and penicillin (100\%) (16). It is not surprising to find this low sensitivity with penicillin and ampicillin. This may be a reflection of pattern of antibiotic use and abuse in the study setting, especially with the common non-prescription use of beta-lactam antibiotics for treatment of many clinical syndromes which encourages resistance. High sensitivity to ceftriaxone and erythromycin may be due to limited exposure of subjects to the prescription antibiotics which are relatively more expensive. Other environmental and socio-economic factors including poor hygiene and purchase of cheap but poor quality of drugs as well as genetic factors may also play key roles in determining pattern of antibiotic sensitivity found in this study $(30,31)$.

\section{Conclusion}

This study revealed that there was a significantly higher prevalence of GBS anogenital colonization among HIV infected subjects. With the high prevalence of HIV especially in the study setting, preventive approach to GBS colonization is a worthy measure to cut down on the complication in the neonates. Level of education has a reverse relationship with rate of colonization, higher in pregnant women with low educational status. There was a marked sensitivity to ceftriaxone and erythromycin but resistance to penicillin $\mathrm{G}$ and ampicillin.

\section{Recommendations}

This high prevalence suggests the need to institute antenatal GBS screening protocol among high risk pregnancies especially among low-literate and HIV positive women. Those that test positive should receive follow-up treatment to prevent feto-maternal adverse effects. There is also, need for improvement in health education and counseling with emphasis on appropriate antibiotic use, personal and environmental hygiene, especially through the antenatal care clinics. There should be concerted effort towards discouraging self-medication and indiscriminate use of antibiotics which may promote drug resistance. This may require strengthening legal and health institutions responsible for pharmacovigilance and surveillance.

\section{Conflict of Interests} None.

\section{Ethical Issues}

Ethical clearance for the study was obtained from the University of Calabar Teaching Hospital Research and Ethics Committee with approval number UCTH/ HREC/1/2015-016 before the commencement of the study.

\section{Financial Support}

None.

\section{References}

1. Elikwu CJ, Oduyebo O, Ogunsola FT, Anorlu RI, Okoromah $\mathrm{CN}$, Konig B. High group B streptococcus carriage rates in pregnant women in a tertiary institution in Nigeria. Pan Afr Med J. 2016;25:249. doi:10.11604/pamj.2016.25.249.9433

2. Clifford V, Garland SM, Grimwood K. Prevention of neonatal group B streptococcus disease in the 21st century. J Paediatr Child Health. 2012;48(9):808-815. doi:10.1111/ j.1440-1754.2011.02203.x

3. Joachim A, Matee MI, Massawe FA, Lyamuya EF. Maternal and neonatal colonisation of group B streptococcus at Muhimbili National Hospital in Dar es Salaam, Tanzania: prevalence, risk factors and antimicrobial resistance. BMC Public Health. 2009;9:437. doi:10.1186/1471-2458-9-437

4. Monyama MC, Bolukaoto JY, Chukwu MO, et al. Group B streptococcus colonisation in pregnant women at Dr. George Mukhari Hospital, South Africa. Southern African Journal of Infectious Diseases. 2016;31(3):74-78. doi:10.108 0/23120053.2016.1156308

5. Paoletti LC, Rench MA, Kasper DL, Molrine D, Ambrosino D, Baker CJ. Effects of alum adjuvant or a booster dose on immunogenicity during clinical trials of group B streptococcal type III conjugate vaccines. Infect Immun. 2001;69(11):6696-6701. doi:10.1128/iai.69.11.66966701.2001

6. Mullaney DM. Group B Streptococcal Infections in Newborns. Journal of Obstetric Gynecologic Neonatal Nursing.2001;30(6):649-658.doi:10.1111/j.1552-6909.2001. tb00012.x

7. Verani JR, McGee L, Schrag SJ. Division of Bacterial Disease, National centre for Immunization and Respiratory Disease Control and Prevention (CDC). Prevention of Perinatal group B streptococcal disease-revised guidelines from CDC, 2010. MMWR Recomm Rep. 2010:59(RR-10):1-36.

8. Heath PT, Balfour G, Weisner AM, et al. Group B streptococcal disease in UK and Irish infants younger than 90 days. Lancet. 2004;363(9405):292-294. doi:10.1016/ s0140-6736(03)15389-5

9. Hassan IA, Onon TS, Weston D, et al. A quantitative descriptive study of the prevalence of carriage (colonisation) of haemolytic streptococci groups A, B, C and G in pregnancy. J Obstet Gynaecol. 2011;31(3):207-209. doi:10. 3109/01443615.2010.541570

10. Saleem S, Rouse DJ, McClure EM, et al. Chlorhexidine vaginal and infant wipes to reduce perinatal mortality and morbidity: a randomized controlled trial. Obstet Gynecol. 2010;115(6):1225-1232. doi:10.1097/ AOG.0b013e3181e00ff0

11. Madhi SA, Radebe K, Crewe-Brown H, et al. High burden of invasive Streptococcus agalactiae disease in South African infants. Ann Trop Paediatr. 2003;23(1):15-23. 
doi:10.1179/000349803125002814

12. Mitima KT, Ntamako S, Birindwa AM, et al. Prevalence of colonization by Streptococcus agalactiae among pregnant women in Bukavu, Democratic Republic of the Congo. J Infect Dev Ctries. 2014;8(9):1195-1200. doi:10.3855/ jidc. 5030

13. Milledge J, Calis JC, Graham SM, et al. Aetiology of neonatal sepsis in Blantyre, Malawi: 1996-2001. Ann Trop Paediatr. 2005;25(2):101-110. doi:10.1179/146532805x45692

14. National Population Commission of Nigeria. Report of the 2006 census and provisional results. http://www.population. gov.ng/index.php.htm. Accessed January 2015.

15. Nwachukwu N, Utsalo S, Kanu I, Anyanwu E. Genital Colonization of Group B Streptococcus at term pregnancy in Calabar, Nigeria. Internet J Pediatr Neonatol. 2006;7(2):14.

16. Onipede A, Adefusi O, Adeyemi A, Adejuyigbe E, Oyelese A, Ogunniyi T. Group B Streptococcus carriage during late pregnancy in Ile-ife, Nigeria. Afr J Clin Exp Microbiol. 2012; 13(3):135-143. doi: 10.4314/ajcem.v13i3.2

17. Donbraye-Emmanuel OB, Okonko ID, Donbraye E, et al. Isolation and characterization of Group B Steptococci and other pathogens among pregnant women in Ibadan Southwest Nigeria. J Appl Biosci. 2010;5902(29):1781-1792.

18. Okon KO, Usman H, Umar Z, Balogun S. Prevalence of Group B Streptoccocus(GBS) colonization among pregnant women attending antenantal clinic of Tertiary Hospital in Northeasten Nigeria. Ame J Res Commun. 2013;1(6):54-61

19. Onwuezobe IA, Effiom RA. Prevalence and associated risk factors of Group B streptococcus in pregnant women attending antenatal care in a Nigerian urban hospital. Ibom Med Jo. 2016;9(1):1-7.

20. Nsagha DS, Bello CS, Kahdakai-Olukemi YT. Maternal Carriage in Pregnancy of Group B Streptococcs in Jos: Relation of Endocervical Anorectal Colonization. Nig Qt J Hosp Med. 1997;7(1):53-56.

21. Uhiara JE. Group B streptococcal carriage among parturients and their neonates in Zaria, Nigeria. Afr J Med Med Sci. 1993;22(3):79-83.

22. Namugongo A, Bazira J, Fajardot Y, Joseph N. Group B Streptococcus Colonization among Pregnant Women Attending Antenatal Care at Tertiary Hospital in Rural
Southwestern Uganda. Int J Microbiol. 2016;2016:3816184. doi:10.1155/2016/3816184

23. Cutland CL, Madhi SA, Zell ER, et al. Chlorhexidine maternal-vaginal and neonate body wipes in sepsis and vertical transmission of pathogenic bacteria in South Africa: a randomised, controlled trial. Lancet. 2009;374(9705):19091916. doi:10.1016/s0140-6736(09)61339-8

24. Tsolia M, Psoma M, Gavrili S, et al. Group B streptococcus colonization of Greek pregnant women and neonates: prevalence, risk factors and serotypes. Clin Microbiol Infect. 2003;9(8):832-838.

25. Narava S, Rajaram G, Ramadevi A, Prakash GV, Mackenzie S. Prevention of perinatal group B streptococcal infections: a review with an Indian perspective. Indian J Med Microbiol. 2014;32(1):6-12. doi:10.4103/0255-0857.124286

26. Dauby N, Chamekh M, Melin P, Slogrove AL, Goetghebuer T. Increased risk of group B streptococcus invasive infection in HIV-exposed but uninfected infants: a review of the evidence and possible mechanisms. Front Immunol. 2016;7:505. doi:10.3389/fimmu.2016.00505

27. El Aila NA, Tency I, Claeys G, et al. Comparison of different sampling techniques and of different culture methods for detection of group B streptococcus carriage in pregnant women. BMC Infect Dis. 2010;10:285. doi:10.1186/14712334-10-285

28. Gray KJ, Kafulafula G, Matemba M, Kamdolozi M, Membe G, French N. Group B Streptococcus and HIV infection in pregnant women, Malawi, 2008-2010. Emerg Infect Dis. 2011;17(10):1932-1935. doi:10.3201/eid1710.102008

29. Shah M, Aziz N, Leva N, Cohan D. Group B Streptococcus colonization by HIV status in pregnant women: prevalence and risk factors. J Womens Health (Larchmt). 2011;20(11):1737-1741. doi:10.1089/jwh.2011.2888

30. Sahoo KC, Tamhankar AJ, Johansson E, Lundborg CS. Antibiotic use, resistance development and environmental factors: a qualitative study among healthcare professionals in Orissa, India. BMC Public Health. 2010;10:629. doi:10.1186/1471-2458-10-629

31. Alekshun MN, Levy SB. Molecular mechanisms of antibacterial multidrug resistance. Cell. 2007;128(6):10371050. doi:10.1016/j.cell.2007.03.004

Copyright ( 2018 The Author (s); This is an open-access article distributed under the terms of the Creative Commons Attribution License (http://creativecommons.org/licenses/by/4.0), which permits unrestricted use, distribution, and reproduction in any medium, provided the original work is properly cited. 